\title{
Expression of Lymphatic Markers in the Adult Rat Spinal Cord
}

\section{OPEN ACCESS}

Edited by:

Francesco Moccia,

University of Pavia, Italy

Reviewed by:

Josef Bischofberger

University of Basel, Switzerland

Aleksanteri Aspelund,

Wihuri Research Institute and University of Helsinki, Finland

*Correspondence:

Falk Schroedl

falk.schroedl@pmu.ac.at

tThese authors have contributed equally to this work and should be considered as last authors.

Received: 09 September 2015 Accepted: 25 January 2016 Published: 09 February 2016

Citation:

Kaser-Eichberger A, Schroedl F, Bieler L, Trost A, Bogner B, Runge $C$ Tempfer H, Zaunmair P, Kreutzer C,

Traweger A, Reitsamer HA and Couillard-Despres S (2016)

Expression of Lymphatic Markers in the Adult Rat Spinal Cord.

Front. Cell. Neurosci. 10:23.

doi: 10.3389/fncel.2016.00023

\begin{abstract}
Alexandra Kaser-Eichberger ${ }^{1}$, Falk Schroed ${ }^{1,2 *}$, Lara Bieler ${ }^{3,4}$, Andrea Trost ${ }^{1}$, Barbara Bogner ${ }^{1}$, Christian Runge ${ }^{1}$, Herbert Tempfer ${ }^{5,6}$, Pia Zaunmair ${ }^{3,4}$, Christina Kreutzer ${ }^{3,4}$, Andreas Traweger ${ }^{5,6}$, Herbert A. Reitsamer ${ }^{1 \dagger}$ and Sebastien Couillard-Despres ${ }^{3,4 t}$

${ }^{1}$ University Clinic of Ophthalmology and Optometry, Research Program for Experimental Ophthalmology and Glaucoma Research, Paracelsus Medical University, Salzburg, Austria, ${ }^{2}$ Institute of Anatomy, Paracelsus Medical University, Salzburg, Austria, ${ }^{3}$ Institute of Experimental Neuroregeneration, Paracelsus Medical University, Salzburg, Austria,

${ }^{4}$ Spinal Cord Injury and Tissue Regeneration Center Salzburg, (SCI-TReCS), Paracelsus Medical University, Salzburg,

Austria, ${ }^{5}$ Institute of Tendon and Bone Regeneration, Paracelsus Medical University, Salzburg, Austria, ${ }^{6}$ Austrian Cluster for Tissue Regeneration, Vienna, Austria
\end{abstract}

Under physiological conditions, lymphatic vessels are thought to be absent from the central nervous system (CNS), although they are widely distributed within the rest of the body. Recent work in the eye, i.e., another organ regarded as alymphatic, revealed numerous cells expressing lymphatic markers. As the latter can be involved in the response to pathological conditions, we addressed the presence of cells expressing lymphatic markers within the spinal cord by immunohistochemistry. Spinal cord of young adult Fisher rats was scrutinized for the co-expression of the lymphatic markers PROX1 and LYVE-1 with the cell type markers Iba1, CD68, PGP9.5, OLIG2. Rat skin served as positive control for the lymphatic markers. PROX1-immunoreactivity was detected in many nuclei throughout the spinal cord white and gray matter. These nuclei showed no association with LYVE-1. Expression of LYVE-1 could only be detected in cells at the spinal cord surface and in cells closely associated with blood vessels. These cells were found to co-express Iba1, a macrophage and microglia marker. Further, double labeling experiments using CD68, another marker found in microglia and macrophages, also displayed co-localization in the lba1+ cells located at the spinal cord surface and those apposed to blood vessels. On the other hand, PROX1-expressing cells found in the parenchyma were lacking lba1 or PGP9.5, but a significant fraction of those cells showed co-expression of the oligodendrocyte lineage marker OLIG2. Intriguingly, following spinal cord injury, LYVE-1-expressing cells assembled and reorganized into putative pre-vessel structures. As expected, the rat skin used as positive controls revealed classical lymphatic vessels, displaying PROX1+ nuclei surrounded by LYVE-1-immunoreactivity. Classical lymphatics were not detected in adult rat spinal cord. Nevertheless, numerous cells expressing either LYVE-1 or PROX1 were identified. Based on their localization and overlapping expression with Iba1, the LYVE-1 + cell population likely represents a macrophage subpopulation, while a significant fraction of PROX1+ cells belong to the oligodendrocytic lineage based on their distribution and the expression of OLIG2. The response of these LYVE-1+ and PROX1+ cell subpopulations to pathological conditions, especially in spinal cord inflammatory conditions, needs to be further elucidated.

Keywords: spinal cord, macrophage, PROX1, LYVE-1, Iba1, OLIG2, lymphatics, spinal cord injury 


\section{INTRODUCTION}

Two crucial roles are carried out by the lymphatic system: (1) fluid homeostasis via the drainage of extracellular fluid; and (2) immune defense through the transport of antigens and immune cells to the lymph nodes (Alitalo, 2011; Card et al., 2014). To fulfill these functions, the lymphatic system is widespread throughout the human body (Breslin, 2014). Nevertheless, a few tissues, such as the (inner) eye (Streilein, 2003) and the central nervous system (CNS; Ransohoff and Engelhardt, 2012), lack a "classical" lymphatic system and are therefore considered to be alymphatic. The epithet "classical" refers to the presence of vessels with a lymphatic phenotype, i.e., with endothelial cells expressing the surface receptors VEGFR3 or LYVE-1 and membrane bound components, such as podoplanin, or transcription- factors, such as PROX1 or FOXC2 (Banerji et al., 1999; Jackson et al., 2001; Sleeman et al., 2001; Jackson, 2007).

Over the last decades, research of the lymphatic system underwent a tremendous boost based on the introduction of these aforementioned markers. While identification of lymphatics within a tissue remains challenging due to the small caliber of the vessels and their structural similarity with small-caliber blood vessels, the markers introduced now allow reliable identification (Sleeman et al., 2001). The crux with lymphatic markers however is that no exclusive marker has been identified so far (Sleeman et al., 2001) since most lymphatic markers are also expressed on cells other than lymphatic endothelium (Matsui et al., 1999; Schroedl et al., 2008). Therefore, for the unequivocal identification of lymphatics, there is a consensus that a combination of several markers is necessary, as recently stated for the inner eye (Schroedl et al., 2014) or under pathological conditions (Van der Auwera et al., 2006). Hence, we chose for this study the combination of the transcription factor PROX1, a homeo-box protein that retains its activity in nuclei of lymphatic endothelium in adulthood (Wigle and Oliver, 1999; Wilting et al., 2002) and LYVE-1, a membrane-bound glycoprotein and one of the best characterized markers of lymphatic endothelium (Jackson, 2004; Baluk and McDonald, 2008).

Being part of the CNS, which is considered to be alymphatic, the existence of structures or cells expressing lymphatic markers has not been thoroughly investigated within the spinal cord. The characterization of these putative lymphatic cells is particularly relevant for repair mechanisms considering that lymphatic cells can organize into a lymphatic system during pathological processes (Paavonen et al., 2000; Kerjaschki et al., 2004; Maruyama et al., 2005; Zumsteg et al., 2009; Tammela and Alitalo, 2010; Kerjaschki, 2014; Tempfer et al., 2015).

In this study, we scrutinize with histological methods the presence of potential lymphatic system or cells expressing lymphatic markers in the healthy, as well as the injured, adult rat spinal cord.

\section{MATERIALS AND METHODS}

\section{Specimens}

All experiments were performed in conformity with the Directive (2010/63/EU) of the European Parliament and of the Council and were approved by the national animal health commission (Land Salzburg, Referat Gesundheitsrecht und Gesundheitsplanung Referat 9/01). Female Fisher 344 rats of approximately 3 months of age were used for this study. For histological analysis, rats received an overdose of anesthetics (ketamine/xylazine/ acepromazine; i.p.) prior to transcardial perfusion with $\mathrm{NaCl}$ $0.9 \%$ followed by phosphate buffered saline (PBS) containing $4 \%$ formaldehyde. The spinal cord and skin samples from the thigh were dissected, further fixed by immersion $(1 \mathrm{~h}$, room temperature, RT) and rinsed in PBS. Spinal cord and skin samples were transferred into PBS containing $15 \%$ sucrose $(12 \mathrm{~h}$ at $4^{\circ} \mathrm{C}$ ), embedded in tissue embedding medium (Slee Technik, Mainz, Germany) and frozen using liquid nitrogen-cooled methylbutane and stored at $-20^{\circ} \mathrm{C}$ until further processing.

\section{Spinal Cord Contusion Injury (SCI)}

Rats were anesthetized using $1.6 \%$ Isoflurane-oxygen mix. For analgesia, $0.03 \mathrm{mg} / \mathrm{kg}$ bodyweight (bw) Buprenorphine (Bupaq ${ }^{\circledR}, 0.3 \mathrm{mg} / \mathrm{mL}$, Richterpharma, Wels, Austria) was injected sub-cutaneous (SC) $30 \mathrm{~min}$ prior to surgery. To prevent hypothermia, body temperature was maintained by a rectal sensor-coupled to a heating pad. Heart frequence and oxygen saturation was monitored throughout the whole surgical procedures (SomnoSuite $\left.{ }^{\circledR}, \mathrm{KENT}\right)$. During surgery, the dorsal aspect of the vertebra at thoracic level 8 was removed to expose the dura mater and the spinal cord. Contusion was performed using an Infinite Horizon impactor (IH-Impactor ${ }^{\circledR}$, Precision Systems and Instrumentation, LLC with a force of $200 \mathrm{kdyn}$ with immediate withdrawal). After that the absence of bleeding has been confirmed, paravertebral muscles were sutured and the skin was closed using a skin stapler. To prevent pain and infections after surgery, $1-2 \mathrm{mg} / \mathrm{kg}$ bw Meloxicam (Metacam ${ }^{\circledR} 5 \mathrm{mg} / \mathrm{ml}$ ) and $10 \mathrm{mg} / \mathrm{kg}$ bw Enrofloxacin (Baytril ${ }^{\circledR} 25 \mathrm{mg} / \mathrm{ml}$ ) was injected SC daily for 5 days. Additionally, $0.01 \mathrm{mg} / \mathrm{kg}$ bw Buprenorphine injected SC twice a day for 2 days post surgery. As bladder function was impaired after SCI, bladder was manually voided 2-3 times per day. Perfusion for histological analysis was performed 14 days post-lesion.

\section{Immunohistochemistry}

Spinal cord and skin samples were sectioned with a cryostat (HM 550, Microm, Walldorf, Germany) in serial sections of $16 \mu \mathrm{m}$, collected on adhesion slides (Superfrost Plus; Thermo Scientific, Wien Austria) and air-dried for $1 \mathrm{~h}$ at RT. Sections were rinsed 5 min in Tris-buffered saline (TBS; Roth, Karlsruhe, Germany) and incubated for $1 \mathrm{~h}$ at RT in TBS containing 5\% donkey serum (Sigma-Aldrich, Wien, Austria), 1\% bovine serum albumin (BSA; Sigma-Aldrich), and 0.5\% Triton X-100 (Merck, Darmstadt, Germany). Sections were then rinsed for $5 \mathrm{~min}$ in TBS and further incubated for single and double immunohistochemistry 
with the primary antibodies (Table 1) diluted in TBS, containing $1 \%$ BSA and $0.5 \%$ Triton X-100, for $12 \mathrm{~h}$ at RT. Sections were rinsed four times for $5 \mathrm{~min}$ in TBS and the primary antibodies visualized by corresponding Alexa488-, or Alexa555conjugated antibodies (Invitrogen, Karlsruhe, Germany) diluted 1:1000 in TBS, containing $1 \%$ BSA and $0.5 \%$ Triton X-100 $(1 \mathrm{~h}$ at $\mathrm{RT})$. Subsequently, the sections were rinsed $5 \mathrm{~min}$ in TBS followed by a 10 min nuclear labeling with $4^{\prime}, 6$ Diamidino-2 phenylindole dihydrochloride (DAPI, $0.25 \mu \mathrm{g} / \mathrm{mL}$; VWR, Vienna, Austria). Finally, sections were rinsed three times for $5 \mathrm{~min}$ in PBS and embedded in TBS-glycerol (1:1 at $\mathrm{pH} 8.6)$.

Rat skin sections were used to validate the LYVE-1 and PROX1 primary antibodies. Additionally, to avoid possible cross-reactivity in experiments involving two or more primary antibodies, successive incubations have been performed (i.e., primary and secondary antibodies for epitope one, followed by incubation with primary and secondary antibodies for epitope two). Negative controls were performed by omission of the primary antibodies during incubation and resulted in absence of immunoreactivity.

\section{Documentation}

Micrographs of immunohistostainings were acquired using a confocal laser scanning unit (Axio ObserverZ1 attached to LSM710, Zeiss, Göttingen, Germany; $\times 20$ dry or $\times 40$ and $\times 60$ oil immersion objektive lenses, with numeric apertures $0.8,1.30$, and 1.4, respectively; Zeiss). All images presented here consist of confocal images in single optical section mode. Negative controls were recorded with identical laser settings as used for documentation of corresponding primary antibodies.

\section{RESULTS}

In the rat adult spinal cord, PROX1-immunoreactivity was detected in numerous nuclei evenly distributed throughout the gray and white matter. Nuclei of the central canal lacked PROX1-immunoreactivity (Figure 1A). Absence of immunoreactivity was observed in the negative controls (Figure 1B), whereas in rat skin, used as positive control, PROX1-positive nuclei were bordering luminal structures (Figure 1C). Detection of the lymphatic endothelial marker LYVE-1 revealed numerous LYVE-1-expressing cells on the surface of the spinal cord, surrounding the ventral spinal artery, and following the sulcal arteries into the anterior median sulcus (Figure 1D). Double labeling experiments demonstrated that cells expressing LYVE-1 did not possess PROX1-positive nuclei (Figure 1D). Corresponding negative controls were lacking immunoreactivity (Figure 1E), whereas controls in rat skin showed cells with PROX-1 positive nuclei surrounded by LYVE-1 immunoreactivity (Figure 1F). Identical results were obtained on the dorsal side of the spinal cord, i.e., LYVE-1immunoreactive cells were detected on the spinal cord surface and also in association with vessels of the dorsal spinal arteries entering the parenchyma (Figures 1G,H). Occasionally, PROX1immunoreactive nuclei were in close vicinity with LYVE-1+ structures, which likely reflected expression of single markers in adjacent cells (Figure 1H). Nevertheless, the overwhelming majority of LYVE-1-positive cells surrounding vessels lacked PROX1 immunoreactivity (Figure 1I).

Double immunohistochemistry with LYVE-1 and Iba1 revealed a co-localization of both markers in cells located at the spinal cord surface (Figure 2A). Within the spinal cord, LYVE-1+/Iba1+ cells surrounding blood vessels were detected, whereas cells within the spinal cord parenchyma solely displayed immunoreactivity for Ibal (Figures 2D,E). Cells displaying immunoreactivity exclusively for LYVE-1 were not observed. Double immunohistochemistry for CD68 and Iba1 revealed an identical pattern, i.e., CD68+/Iba1+ cells were detected on the spinal cord surface (Figure 2F), following the spinal cord arteries (Figures 2F,G). Cells immunoreactive exclusively for CD68 were not observed. Corresponding negative controls ascertained the absence of immunoreactivity (Figure 2B). In contrast, in the rat skin positive controls, two distinct cell populations were observed expressing either LYVE-1 or Ibal without apparent co-localization (Figure 2C).

To further characterize the PROX1+ cells within the spinal cord, PROX1-immunohistochemistry was combined with either Ibal, the pan-neuronal marker PGP9.5, or the oligodendrocyte lineage marker OLIG2. These double labeling experiments revealed that PROX1+ nuclei were not associated with Iba1 (Figure 3A), while corresponding controls showed absence of immunoreactivity (Figure 3B). Similarly, PGP9.5-immunoreactivity was not associated with PROX1+ nuclei in both small- and large-sized neurons (Figure 3C). Corresponding negative controls were not immunoreactive (Figure 3D). In contrast, the combination of PROX1 and OLIG2 revealed extensive co-localization of both markers in nuclei throughout the spinal cord

TABLE 1 | Primary antibodies used in this study

\begin{tabular}{llll}
\hline Markers & Symbols & Hosts & Company \\
\hline Prospero homeobox protein 1 & PROX1 & Mouse & Acris; Herford, Germany \\
Lymphatic vessel endothelial hyaluronan receptor 1 & LYVE-1 & Rabbit & Acris; Herford, Germany \\
Cluster of differentiation 68 & CD68 & Rabbit & Abcam; Cambridge, UK \\
lonized calcium-binding adapter molecule 1 & Iba1 & Goat & Wako Chemicals; Neuss, Germany \\
Protein-gene product 9.5 & PGP9.5 & Guinea pig & Merck Millipore; Vienna, Austria \\
Oligodendrocyte transcription factor 2 & OLIG2 & Rabbit & Merck Millipore; Vienna, Austria \\
Major histocompatibility complex class II & MHCII & Mouse & Abcam; Cambridge, UK
\end{tabular}




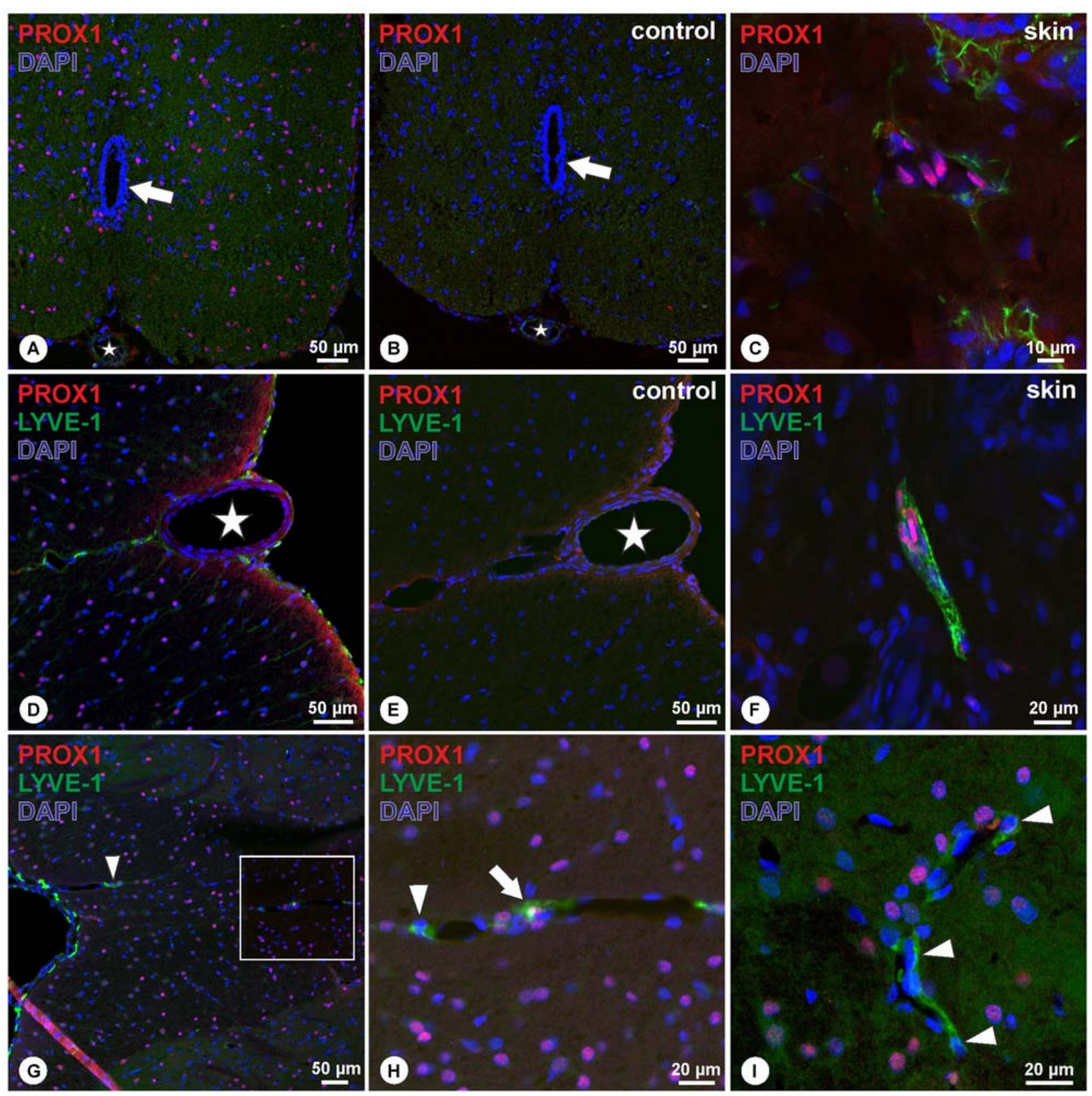

FIGURE 1 | (A-C) In cross sections of the spinal cord, PROX1-immunoreactivity (red) was detected in numerous nuclei (DAPI, blue) throughout the gray and white matter without preference, as indicated by purple mixed color (A), whereas negative controls revealed absence of immunoreactivity (B). Asterisk (A,B) indicates ventral spinal artery, arrow (A,B) indicates the central canal. Controls in cross sections of rat skin (C) revealed nuclear Prox1 immunoreactivity as in (A). (D-F) Double-immunohistochemistry of PROX1 (red) and LYVE-1 (green) reveals LYVE-1+ cells lacking PROX1-immunoreactivity on the surface of the spinal cord and following the anterior sulcal arteries (D). Immunoreactivity was absent in negative controls (E). Asterisk in (D,E) indicates ventral spinal artery. Skin controls revealed PROX1+ nuclei surrounded by LYVE-1-immunoreactivity (F). Blue: DAPI. (G-I) Double-immunohistochemistry of PROX1 (red) and LYVE-1 (green) reveals LYVE-1+ cells on the surface of the posterior spinal cord lacking PROX1-immunoreactivity (G), following the posterior spinal arteries (arrowhead). While occasionally cells were detected with apparent association of PROX1 and LYVE-1 (arrow in $\mathbf{H}$ ), the majority of cells displayed immunoreactivity for LYVE-1 only (arrowheads in $\mathbf{H , I})$. (H) represents magnification of the boxed area in $(\mathbf{G})$. Blue: DAPI.

(Figure 3E). Characterization in five randomly chosen micrographs of approximately 200 nuclei expressing either marker revealed that $46 \%$ were PROX1+/OLIG2+, whereas $42 \%$ showed immunoreactivity for OLIG2 only and $12 \%$ displayed immunoreactivity for PROX1 only. PROX1/OLIG2 immunoreactivity was absent in corresponding negative controls (Figure 3F).

Preliminary investigation in lesioned spinal cord revealed cell debris and cells accumulation within the lesion site. Many cells displayed immunoreactivity for CD68 (Figure 4A), while 

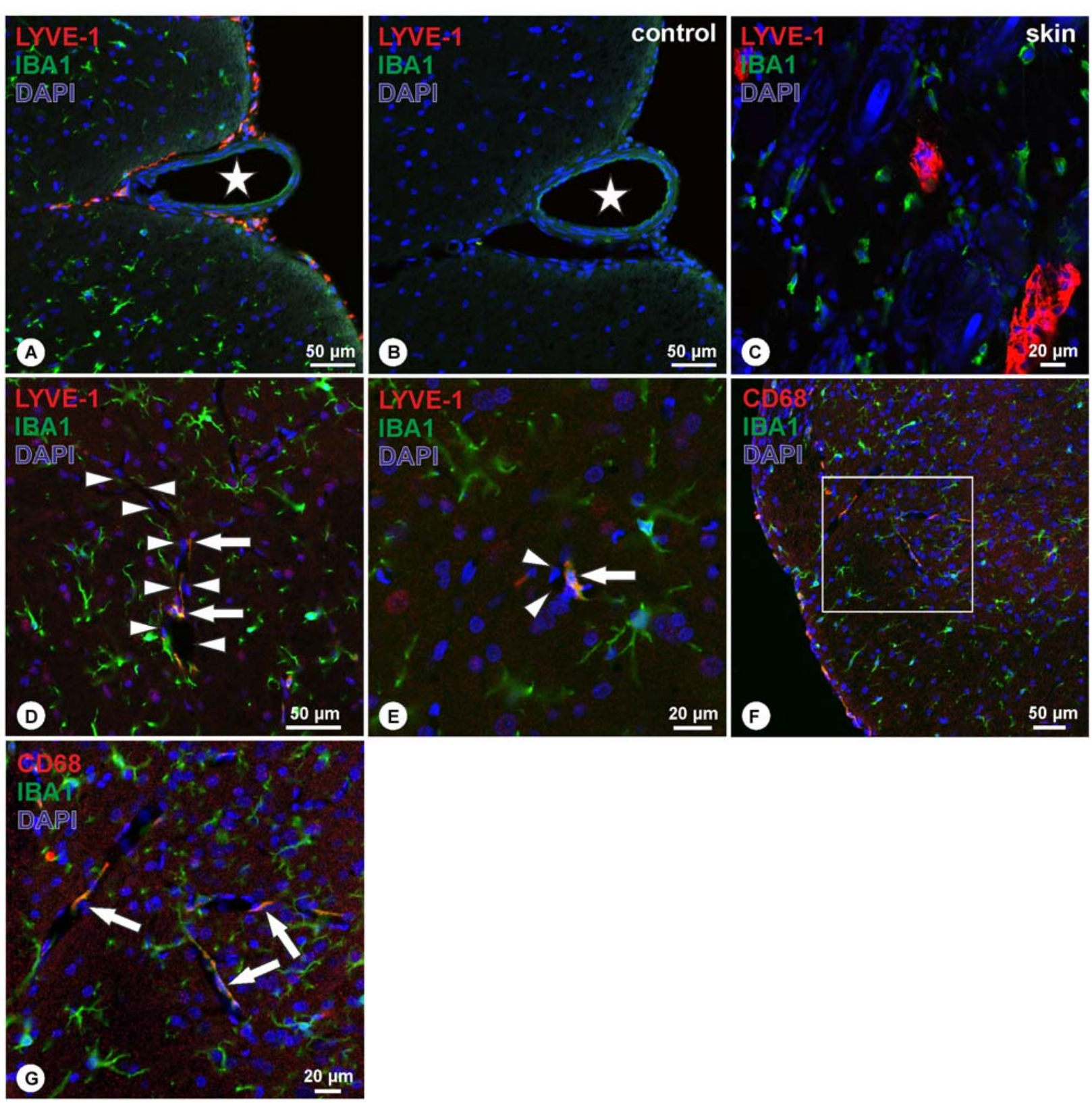

FIGURE 2 | (A-C) Double-immunohistochemistry of LYVE-1 (red) and lba1 (green) reveals LYVE-1+/lba1+ cells on the surface of the spinal cord, following the anterior sulcal arteries (A), whereas negative controls revealed absence of immunoreactivity $\mathbf{( B )}$. Asterisk in $\mathbf{( A , B )}$ indicates ventral spinal artery. Skin controls revealed DAPI+ nuclei (blue) surrounded by LYVE-1-immunoractivity (C), and dispersed cells showing immunoreactivity for Iba1 only. (D,E) Within the spinal cord parenchyma, double immunohistochemistry for LYVE-1 (red) and lba1 (green) reveals cells co-localizing for both markers (arrows in D,E) bordering spinal cord blood vessels (arrowheads in D,E), whereas the majority of cells displayed immunoreactivity for Iba1 only. Blue: DAPI. (F,G) Double-immunohistochemistry of CD68 (red) and lba1 (green) reveals CD68+/lba1+ cells on the surface of the spinal cord $\mathbf{( F )}$, and on cells bordering spinal cord blood vessels (arrows in $\mathbf{G})$. (G) Higher magnification of boxed area in $\mathbf{( F )}$. Blue: DAPI.

a subpopulation of CD68+ cells was also immunoreactive for MCHII (Figure 4A). Iba1-immunoreactive cells were interspersed between CD68+/MHCII+ cells (Figure 4A). The amount of CD68-immunoreactive cells decreases towards the periphery of the lesion site, and similarly Iba1+ cells were less frequently detected. Within the lesion site, LYVE-1+ cells assembled in structures that were not observed in the unlesioned tissue (Figures $\mathbf{4 B}, \mathbf{C}$ ). These LYVE-1+ structures appeared organized (Figure 4C), where associated with elongated nuclei, and displayed vessel-like formation (Figures 4C,D). Double immunohistochemistry with LYVE-1 and PROX1 revealed that the majority of LYVE-1 immunoreactive structures were not associated with PROX1-poitive nuclei (Figure 4E). However, in few 

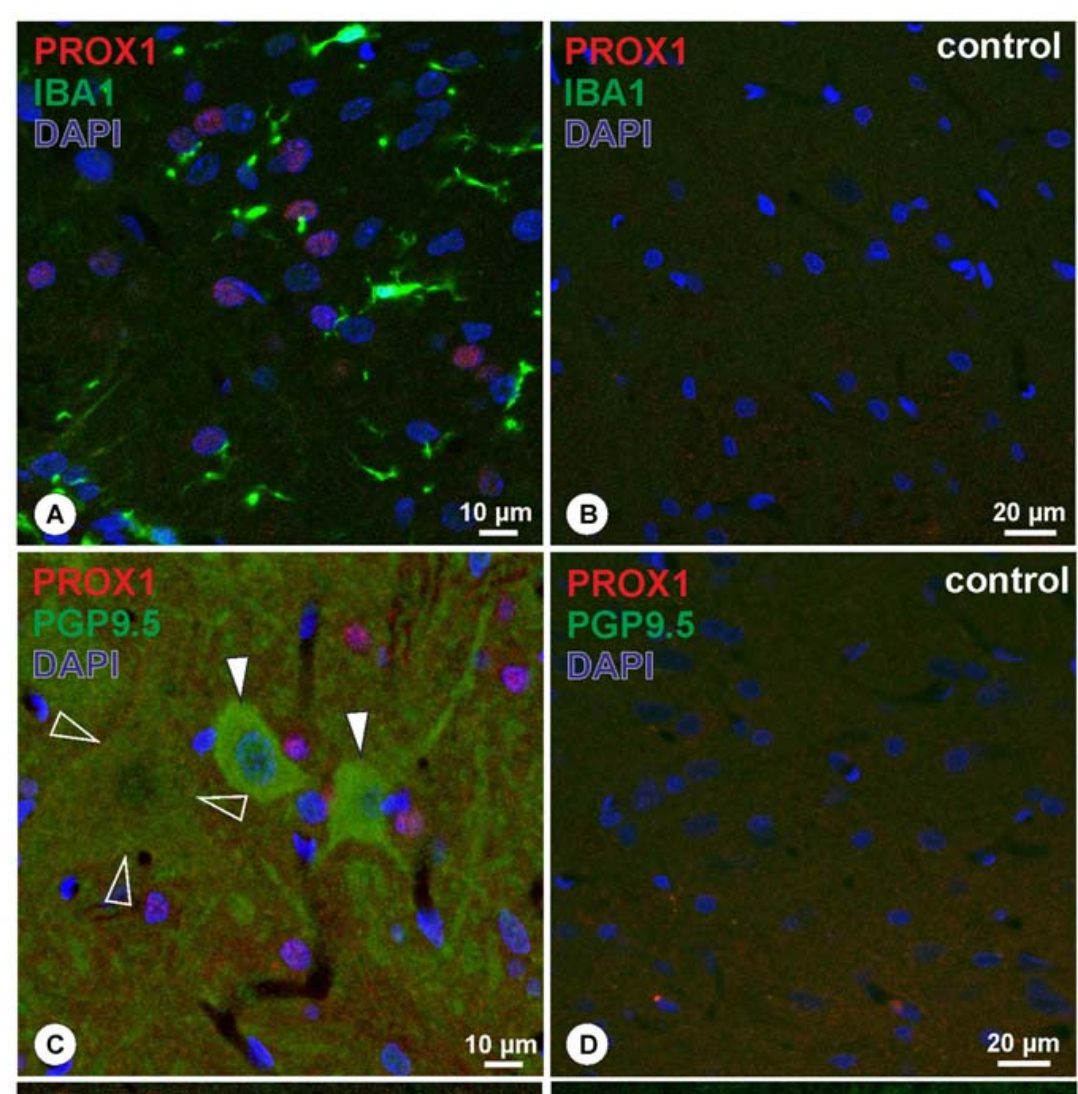

PROX1

control

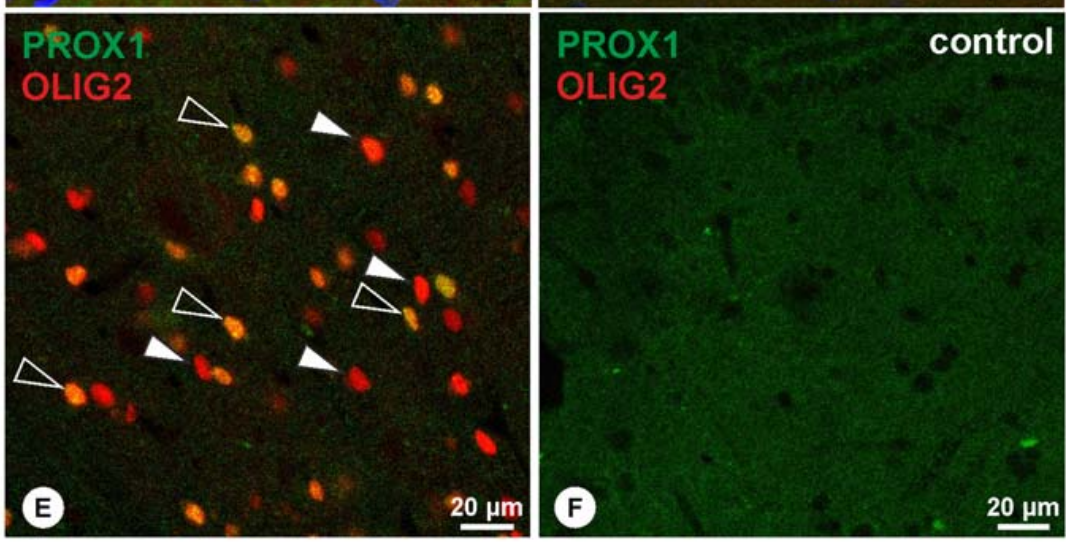

FIGURE 3 | (A,B) Double-immunohistochemistry of PROX1 (red) and lba1 (green) reveals that PROX1 positive nuclei (purple mixed color) are not associated with Iba1-positive cells (A), whereas negative controls lack immunoreactivity (B). Blue: DAPI. (C,D) Double-immunohistochemistry of PROX1 (red) and the pan-neuronal marker PGP9.5 (green) reveals that PROX1-positive nuclei (purple mixed color) are not associated with neurons of the spinal cord (C; arrowheads point to small neurons, open arrowheads outline a faint immunoreactive large neuron), whereas negative controls lack immunoreactivity (D). Blue: DAPI. (E,F) Doubleimmunohistochemistry of PROX1 (green) and OLIG2 (red) reveals co-localized nuclei (E; yellow mixed color, open arrowheads), whereas some nuclei display OLIG2-immunoreactivity only (E; arrowheads). Immunoreactivity was absent in corresponding negative controls (F).

instances, PROX1-positive nuclei were closely related to LYVE-1 immunoreactive structures (Figure 4F).

\section{DISCUSSION}

The dominant opinion in current textbooks considers the CNS as an alymphatic environment (Iliff and Nedergaard, 2013).
However, with the availability of lymphatic markers, this dogma has been recently revisited and challenged (Aspelund et al., 2015; Louveau et al., 2015; Wood, 2015). Therefore, we investigated adult rat spinal cord for the expression of components specific for lymphatic endothelium, namely the membrane bound glycoprotein LYVE-1 in combination with the transcription factor PROX1. LYVE-1 as a marker 

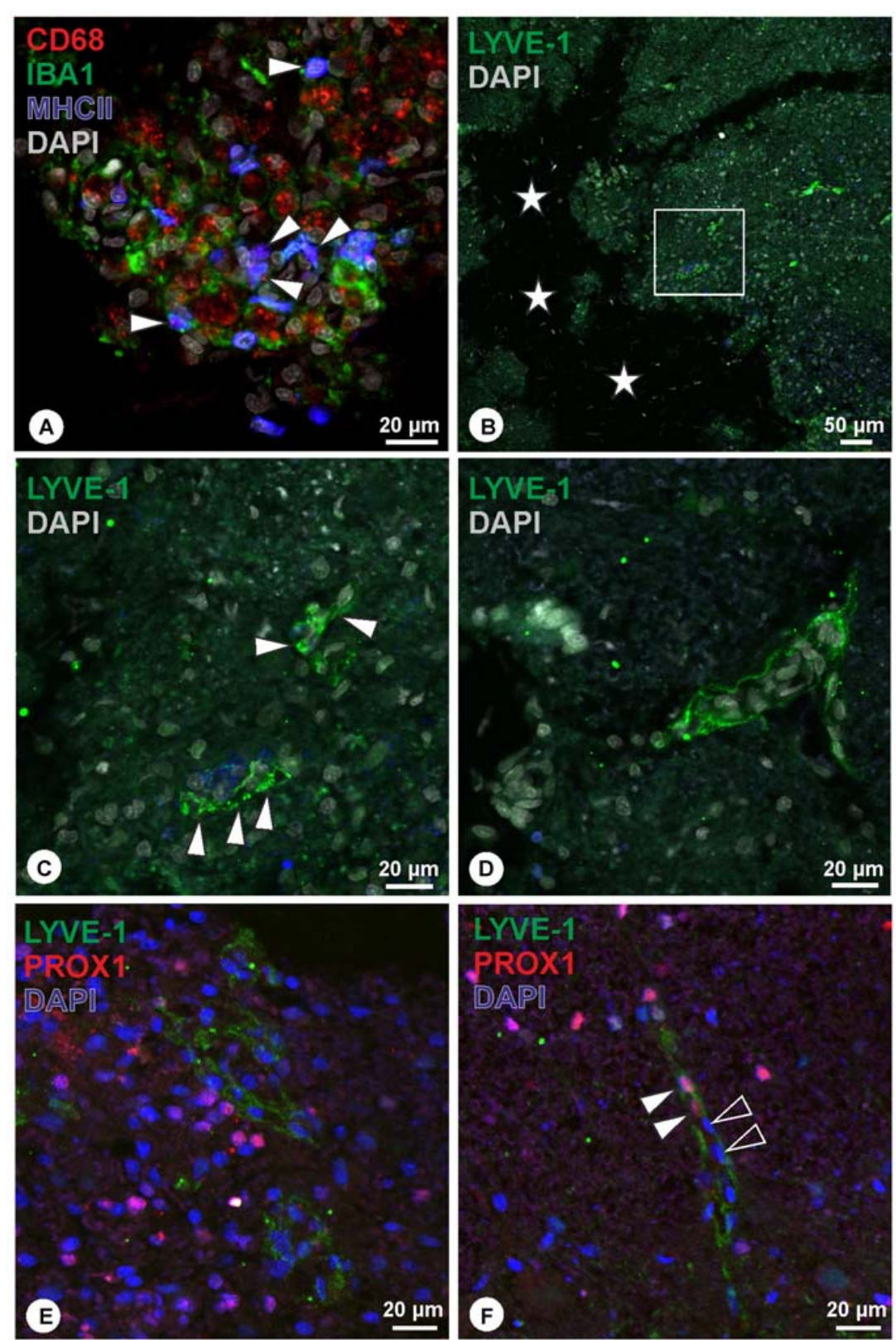

FIGURE 4 | (A) Within the lesion site, many cells were detected displaying immunoreactivity for CD68 (red), a subpopulation of which co-localized for MHCll (blue, arrowheads), while lba1-immunoreactive cells (green) were intermingling. (B,C) Close to the lesion center (asterisks), organized LYVE-1 immunoreactive structures (green) were detected (C, arrowheads; magnification of boxed area in B), displaying vessel-like appearance. DAPI: gray. (D) Another example of vessel-like structure expressing LYVE-1. DAPI: gray. (E,F) Double immunohistochemistry of LYVE-1 (green) and PROX1 (red) revealed that the majority of LYVE-1 immunoreactive structures were not associated with PROX1-positive nuclei (E). In few instances, PROX1 positive nuclei were detected closely associated with LYVE-1 immunoreactive structures (F, arrowheads), while other nuclei in proximity were lacking PROX1 (F, open arrowheads).

for lymphatic endothelium was first identified in 1999 by Banerji et al. (1999). However, its expression is not exclusive for lymphatics, since it is also detected among others on liver sinusoids (Mouta Carreira et al., 2001), pulmonary (Favre et al., 2003) and renal glomerular capillaries (Lee et al., 2011) and its expression pattern may also change along the lymphatic vascular tree (Baluk and McDonald, 2008).
The function of LYVE-1 in cells of these various systems is still not fully understood (Jackson, 2009). On the other side, PROX1 is a homeobox transcription factor and a key player in the development of many organ systems, such as the enterohepatic system or heart (Oliver et al., 1993; Sosa-Pineda et al., 2000). In the CNS, PROX1 is known as a critical regulator of neurogenesis and neuronal differentiation (reviewed in Stergiopoulos et al., 2014). In nuclei of lymphatic endothelial cells, PROX1 expression 
persists into adulthood in physiological as well as in pathological conditions (Wilting et al., 2002). Here, PROX1 is considered to be a master gene (Hong and Detmar, 2003) in lymphatic endothelial progenitor cells controlling the expression of other lymphatic markers (for review, see Yang and Oliver, 2014).

Although we could readily detect these two markers closely associated in lymphatic endothelial cells of the skin, our lymphatic control tissue, our study demonstrated the absence of PROX1+/LYVE-1+ cells in the spinal cord. On the other hand, we detected numerous cells expressing either of these markers, however, under physiological conditions, these were not associated with lymphatic vessel-like structures. The LYVE-1+ cells were found at the surface of the spinal cord or closely located to spinal cord blood vessels. These LYVE+ cells co-localized with the macrophage and microglia markers Iba1, and most likely also CD68, as observed by the almost overlap of the latter with Iba1-immunoreactivity. This marker combination, together with the localization in vicinity to blood vessels highly suggests that this cell population represents macrophages.

Indeed, brain macrophages are mainly situated close to cerebral blood vessels under physiological conditions (Bogie et al., 2014) and LYVE-1 expression in macrophages is well established in other systems (Schledzewski et al., 2006; Cho et al., 2007; Schroedl et al., 2008). While macrophages play an important role during pathological processes (Shechter and Schwartz, 2013), the role of the LYVE-1+ macrophage subpopulation in the spinal cord in physiological and pathological conditions remains to be elucidated. Following spinal cord contusion injury, we could detect a re-organization and redistribution of cells expressing LYVE-1 within the lesioned parenchyma. Some of these cells reorganized in structures resembling putative pre-vessels (Figure 4). Therefore, following lesion, there is a solid evidence to reconsider the possibility of lymphatics within the spinal cord, and most likely also within the brain.

PROX1+ cells were found widespread throughout the spinal cord parenchyma. These PROX1-positive nuclei were neither detected in association with the microglia/macrophage marker Iba1, nor with the pan-neuronal marker PGP9.5, thus ruling out that they represented microglia or neurons, respectively. Instead, PROX1+ nuclei were co-localized with the oligodendrocyte lineage marker OLIG2. The co-existence of these two markers within a cell population is particularly puzzling since Olig2 and PROX1 have been reported to reciprocally suppress the expression of each other during development and in forcedexpression experiments (Kaltezioti et al., 2014; Stergiopoulos et al., 2014). Nevertheless, in a study addressing the gene

\section{REFERENCES}

Alitalo, K. (2011). The lymphatic vasculature in disease. Nat. Med. 17, 1371-1380. doi: $10.1038 / \mathrm{nm} .2545$

Aspelund, A., Antila, S., Proulx, S. T., Karlsen, T. V., Karaman, S., Detmar, M., et al. (2015). A dural lymphatic vascular system that drains brain interstitial expression pattern found in the various cell populations of the CNS, Cahoy et al. (2008) found that Olig2 and PROX1 are enriched in the oligodendroglial lineage. Moreover, the expression of PROX1 was five times higher in myelin-producing oligodendrocytes as compared to oligodendrocyte precursor cells (OPCs). Hence, the PROX1+/Olig2+ cell population detected in the spinal cord probably consists of mature oligodendrocytes. Whether the small percentage of cells expressing solely PROX1 constitutes a distinct population, or a population with low Olig2 expression, remains to be deciphered.

With this study, we confirmed the assumption that classical lymphatic vessels are absent from the healthy adult rat spinal cord. Nevertheless, recent data generated under pathological conditions suggested the development of lymphatic structures in otherwise alymphatic environments, as seen for example in cornea (Cursiefen et al., 2002) or tendon lesions (Tempfer et al., 2015). On the other hand, the existence of structures capable of fulfilling lymphatic vessel-like functions, as recently described for e.g., the Schlemm's Canal of the eye (Aspelund et al., 2014), remains to be demonstrated in the spinal cord. The response of the LYVE-1 positive macrophages observed following spinal cord lesion needs to be further investigated as they may be the prerequisite for the formation of lymphatic-like structures (Maruyama et al., 2005; Alitalo, 2011; Ran and Montgomery, 2012; Kerjaschki, 2014).

\section{AUTHOR CONTRIBUTIONS}

FS, AK-E, SC-D, HAR conceived and designed the study and AK-E, FS, CK, LB, PZ performed the experiments or contributed to data acquisition. FS, AK-E, CK, LB, SC-D analyzed the data and AT, PZ, CK, HT, BB, CR contributed to data interpretation. AK-E, FS, SC-D wrote the manuscript and AK-E, FS, SC-D, HAR, HT, AT, PZ, $\mathrm{LB}, \mathrm{CK}, \mathrm{AT}, \mathrm{BB}, \mathrm{CR}$ critically reviewed the manuscript. All authors read and approved the final version of the manuscript.

\section{ACKNOWLEDGMENTS}

This study was supported by grants from the Research Fund of the Paracelsus Medical University (R13-/01/042KAS, R15/02/067-KAS (AK-E); E-14/20/107-TEM (HT), and R-13/05/054-GRA (LB)), the Austrian Science Fund FWF Special Research Program (SFB) F44 (F4413-B23) "Cell Signaling in Chronic CNS Disorders", The Lotte Schwarz Endowment for Experimental Ophthalmology and Glaucoma Research, The Fuchs-Foundation for Research in Ophthalmology.

fluid and macromolecules. J. Exp. Med. 212, 991-999. doi: 10.1084/jem. 20142290

Aspelund, A., Tammela, T., Antila, S., Nurmi, H., Leppänen, V. M., Zarkada, G., et al. (2014). The Schlemm's canal is a VEGF-C/VEGFR-3responsive lymphatic-like vessel. J. Clin. Invest. 124, 3975-3986. doi: 10.1172/ JCI75395 
Baluk, P., and McDonald, D. M. (2008). Markers for microscopic imaging of lymphangiogenesis and angiogenesis. Ann. N Y Acad. Sci. 1131, 1-12. doi: 10. 1196/annals.1413.001

Banerji, S., Ni, J., Wang, S. X., Clasper, S., Su, J., Tammi, R., et al. (1999). LYVE-1, a new homologue of the CD44 glycoprotein, is a lymphspecific receptor for hyaluronan. J. Cell Biol. 144, 789-801. doi: 10.1083/jcb. 144.4.789

Bogie, J. F., Stinissen, P., and Hendriks, J. J. (2014). Macrophage subsets and microglia in multiple sclerosis. Acta Neuropathol. 128, 191-213. doi: 10. 1007/s00401-014-1310-2

Breslin, J. W. (2014). Mechanical forces and lymphatic transport. Microvasc. Res. 96, 46-54. doi: 10.1016/j.mvr.2014.07.013

Cahoy, J. D., Emery, B., Kaushal, A., Foo, L. C., Zamanian, J. L., Christopherson, K. S., et al. (2008). A transcriptome database for astrocytes, neurons and oligodendrocytes: a new resource for understanding brain development and function. J. Neurosci. 28, 264-278. doi: 10.1523/JNEUROSCI. 4178-07.2008

Card, C. M., Yu, S. S., and Swartz, M. A. (2014). Emerging roles of lymphatic endothelium in regulating adaptive immunity. J. Clin. Invest. 124, 943-952. doi: 10.1172/JCI73316

Cho, C. H., Koh, Y. J., Han, J., Sung, H. K., Jong Lee, H., Morisada, T., et al. (2007). Angiogenic role of LYVE-1-positive macrophages in adipose tissue. Circ. Res. 100, e47-e57. doi: 10.1161/01.res.0000259564.92792.93

Cursiefen, C., Schlötzer-Schrehardt, U., Küchle, M., Sorokin, L., BreitenederGeleff, S., Alitalo, K., et al. (2002). Lymphatic vessels in vascularized human corneas: immunohistochemical investigation using LYVE-1 and podoplanin. Invest. Ophthalmol. Vis. Sci. 43, 2127-2135.

Favre, C. J., Mancuso, M., Maas, K., McLean, J. W., Baluk, P., and McDonald, D. M. (2003). Expression of genes involved in vascular development and angiogenesis in endothelial cells of adult lung. Am. J. Physiol. Heart Circ. Physiol. 285, H1917-H1938. doi: 10.1152/ajpheart.00983.2002

Hong, Y. K., and Detmar, M. (2003). Prox1, master regulator of the lymphatic vasculature phenotype. Cell Tissue Res. 314, 85-92. doi: 10.1007/s00441-0030747-8

Iliff, J. J., and Nedergaard, M. (2013). Is there a cerebral lymphatic system? Stroke 44, S93-S95. doi: 10.1161/STROKEAHA.112.678698

Jackson, D. G. (2004). Biology of the lymphatic marker LYVE-1 and applications in research into lymphatic trafficking and lymphangiogenesis. APMIS 112, 526-538. doi: 10.1111/j.1600-0463.2004.apm11207-0811.x

Jackson, D. G. (2007). Lymphatic markers, tumour lymphangiogenesis and lymph node metastasis. Cancer Treat. Res. 135, 39-53. doi: 10.1007/978-0-38769219-7_4

Jackson, D. G. (2009). Immunological functions of hyaluronan and its receptors in the lymphatics. Immunol. Rev. 230, 216-231. doi: 10.1111/j.1600-065X.2009. 00803.x

Jackson, D. G., Prevo, R., Clasper, S., and Banerji, S. (2001). LYVE-1, the lymphatic system and tumor lymphangiogenesis. Trends Immunol. 22, 317-321. doi: 10. 1016/s1471-4906(01)01936-6

Kaltezioti, V., Antoniou, D., Stergiopoulos, A., Rozani, I., Rohrer, H., and Politis, P. K. (2014). Prox1 regulates Olig2 expression to modulate binary fate decisions in spinal cord neurons. J. Neurosci. 34, 15816-15831. doi: 10. 1523/JNEUROSCI.1865-14.2014

Kerjaschki, D. (2014). The lymphatic vasculature revisited. J. Clin. Invest. 124, 874-877. doi: 10.1172/JCI74854

Kerjaschki, D., Regele, H. M., Moosberger, I., Nagy-Bojarski, K., Watschinger, B., Soleiman, A., et al. (2004). Lymphatic neoangiogenesis in human kidney transplants is associated with immunologically active lymphocytic infiltrates. J. Am. Soc. Nephrol. 15, 603-612. doi: 10.1097/01.asn.0000113316. $52371.2 \mathrm{e}$

Lee, H. W., Qin, Y. X., Kim, Y. M., Park, E. Y., Hwang, J. S., Huo, G. H., et al. (2011). Expression of lymphatic endothelium-specific hyaluronan receptor LYVE-1 in the developing mouse kidney. Cell Tissue Res. 343, 429-444. doi: 10. 1007/s00441-010-1098-x

Louveau, A., Smirnov, I., Keyes, T. J., Eccles, J. D., Rouhani, S. J., Peske, J. D., et al. (2015). Structural and functional features of central nervous system lymphatic vessels. Nature 523, 337-341. doi: 10.1038/nature14432

Maruyama, K., Ii, M., Cursiefen, C., Jackson, D. G., Keino, H., Tomita, M., et al. (2005). Inflammation-induced lymphangiogenesis in the cornea arises from CD11b-positive macrophages. J. Clin. Invest. 115, 2363-2372. doi: 10. $1172 /$ jci23874

Matsui, K., Breitender-Geleff, S., Soleiman, A., Kowalski, H., and Kerjaschki, D. (1999). Podoplanin, a novel 43-kDa membrane protein, controls the shape of podocytes. Nephrol. Dial. Transplant. 14, 9-11. doi: 10.1093/ndt/14. suppl_1.9

Mouta Carreira, C., Nasser, S. M., di Tomaso, E., Padera, T. P., Boucher, Y., Tomarev, S. I., et al. (2001). LYVE-1 is not restricted to the lymph vessels: expression in normal liver blood sinusoids and down-regulation in human liver cancer and cirrhosis. Cancer Res. 61, 8079-8084.

Oliver, G., Sosa-Pineda, B., Geisendorf, S., Spana, E. P., Doe, C. Q., and Gruss, P. (1993). Prox 1, a prospero-related homeobox gene expressed during mouse development. Mech. Dev. 44, 3-16. doi: 10.1016/0925-4773(93) 90012-m

Paavonen, K., Puolakkainen, P., Jussila, L., Jahkola, T., and Alitalo, K. (2000). Vascular endothelial growth factor receptor-3 in lymphangiogenesis in wound healing. Am. J. Pathol. 156, 1499-1504. doi: 10.1016/s0002-9440(10) 65021-3

Ran, S., and Montgomery, K. E. (2012). Macrophage-mediated lymphangiogenesis: the emerging role of macrophages as lymphatic endothelial progenitors. Cancers (Basel) 4, 618-657. doi: 10.3390/cancers40 30618

Ransohoff, R. M., and Engelhardt, B. (2012). The anatomical and cellular basis of immune surveillance in the central nervous system. Nat. Rev. Immunol. 12, 623-635. doi: 10.1038/nri3265

Schledzewski, K., Falkowski, M., Moldenhauer, G., Metharom, P., Kzhyshkowska, J., Ganss, R., et al. (2006). Lymphatic endothelium-specific hyaluronan receptor LYVE-1 is expressed by stabilin-1+, F4/80+, CD11b+ macrophages in malignant tumours and wound healing tissue in vivo and in bone marrow cultures in vitro: implications for the assessment of lymphangiogenesis. J. Pathol. 209, 67-77. doi: 10.1002/path.1942

Schroedl, F., Brehmer, A., Neuhuber, W. L., Kruse, F. E., May, C. A., and Cursiefen, C. (2008). The normal human choroid is endowed with a significant number of lymphatic vessel endothelial hyaluronate receptor 1 (LYVE-1)positive macrophages. Invest. Ophthalmol. Vis. Sci. 49, 5222-5229. doi: 10. 1167/iovs.08-1721

Schroedl, F., Kaser-Eichberger, A., Schlereth, S. L., Bock, F., Regenfuss, B., Reitsamer, H. A., et al. (2014). Consensus statement on the immunohistochemical detection of ocular lymphatic vessels. Invest. Ophthalmol. Vis. Sci. 55, 6440-6442. doi: 10.1167/iovs.14-15638

Shechter, R., and Schwartz, M. (2013). Harnessing monocyte-derived macrophages to control central nervous system pathologies: no longer 'if but 'how'. J. Pathol. 229, 332-346. doi: 10.1002/path.4106

Sleeman, J. P., Krishnan, J., Kirkin, V., and Baumann, P. (2001). Markers for the lymphatic endothelium: in search of the holy grail? Microsc. Res. Tech. 55, 61-69. doi: 10.1002/jemt.1157

Sosa-Pineda, B., Wigle, J. T., and Oliver, G. (2000). Hepatocyte migration during liver development requires Prox1. Nat. Genet. 25, 254-255. doi: 10.1038/ 76996

Stergiopoulos, A., Elkouris, M., and Politis, P. K. (2014). Prospero-related homeobox 1 (Prox1) at the crossroads of diverse pathways during adult neural fate specification. Front. Cell. Neurosci. 8:454. doi: 10.3389/fncel.2014. 00454

Streilein, J. W. (2003). Ocular immune privilege: the eye takes a dim but practical view of immunity and inflammation. J. Leukoc. Biol. 74, 179-185. doi: 10. $1189 /$ jlb.1102574

Tammela, T., and Alitalo, K. (2010). Lymphangiogenesis: molecular mechanisms and future promise. Cell 140, 460-476. doi: 10.1016/j.cell.2010.01.045

Tempfer, H., Kaser-Eichberger, A., Korntner, S., Lehner, C., Kunkel, N., Traweger, A., et al. (2015). Presence of lymphatics in a rat tendon lesion model. Histochem. Cell Biol. 143, 411-419. doi: 10.1007/s00418-014-1287-x

Van der Auwera, I., Cao, Y., Tille, J. C., Pepper, M. S., Jackson, D. G., Fox, S. B., et al. (2006). First international consensus on the methodology of lymphangiogenesis quantification in solid human tumours. Br. J. Cancer 95, 1611-1625. doi: 10.1038/sj.bjc.6603445

Wigle, J. T., and Oliver, G. (1999). Prox1 function is required for the development of the murine lymphatic system. Cell 98, 769-778. doi: 10.1016/s00928674(00)81511-1 
Wilting, J., Papoutsi, M., Christ, B., Nicolaides, K. H., von Kaisenberg, C. S., Borges, J., et al. (2002). The transcription factor Proxl is a marker for lymphatic endothelial cells in normal and diseased human tissues. FASEB J. 16, 1271-1273. doi: 10.1096/fj.01-1010fje

Wood, H. (2015). Neuroimmunology: uncovering the secrets of the 'brain drain'the CNS lymphatic system is finally revealed. Nat. Rev. Neurol. 11:367. doi: 10. 1038/nrneurol.2015.105

Yang, Y., and Oliver, G. (2014). Development of the mammalian lymphatic vasculature. J. Clin. Invest. 124, 888-897. doi: 10.1172/JCI71609

Zumsteg, A., Baeriswyl, V., Imaizumi, N., Schwendener, R., Rüegg, C., and Christofori, G. (2009). Myeloid cells contribute to tumor lymphangiogenesis. PLoS One 4:e7067. doi: 10.1371/journal.pone.0007067
Conflict of Interest Statement: The authors declare that the research was conducted in the absence of any commercial or financial relationships that could be construed as a potential conflict of interest.

Copyright (C) 2016 Kaser-Eichberger, Schroedl, Bieler, Trost, Bogner, Runge, Tempfer, Zaunmair, Kreutzer, Traweger, Reitsamer and Couillard-Despres. This is an openaccess article distributed under the terms of the Creative Commons Attribution License (CC BY). The use, distribution and reproduction in other forums is permitted, provided the original author(s) or licensor are credited and that the original publication in this journal is cited, in accordance with accepted academic practice. No use, distribution or reproduction is permitted which does not comply with these terms. 